\title{
The Effect of Force Feedback on Remote Palpation
}

\author{
R. L. Feller, C. K. L. Lau, C. R. Wagner, D. P. Perrin, R. D. Howe \\ Harvard University \\ Division of Engineering and Applied Sciences \\ Cambridge, Massachusetts \\ \{feller, lau, cwagner\}@ fas.harvard.edu; \{dperrin, howe\}@deas.harvard.edu
}

\begin{abstract}
Combining teletaction systems with telemanipulation systems promises to enhance task performance when interacting with remote environments. However, the force scaling inherent in the telemanipulation system affects the ability of the user to control the exploration force. The quality of the tactile signal is therefore impacted, affecting performance in tasks that benefit from spatially distributed force information. We compare performance localizing an embedded lump in a compliant environment using a telemanipulated teletaction system versus a directly manipulated teletaction system. Lump localization accuracy was found to be the same; however, time required to localize the lump was up to $150 \%$ longer for the telemanipulation trials. Based upon our results, we conclude that the ability to maintain an appropriate force in the remote enviroment is necessary to take full advantage of the spatially distributed force information from the tactile sensor.
\end{abstract}

\section{INTRODUCTION}

Haptic feedback in telemanipulation attempts to simulate the sensation of direct contact with a remote environment. Most current systems only relay a vector of force information for each contact point. These telemanipulators can be enhanced with the transmission of spatially distributed force information [1]. Such "teletaction" systems incorporate tactile sensors that record spatial pressure or shape profiles with tactile displays that recreate the corresponding pressure or shape distribution against the operator's finger pads. The presence of spatially distributed forces has been shown to significantly enhance spatial acuity, orientation detection, and performance in lump detection tasks using palpation [2]. Combining a telemanipulation system with a teletaction system allows the user to control the tactile sensor position and exploration force in a remote environment. Such a system will be valuable in tasks involved in remote medicine and minimally invasive surgery, where the addition of tactile feedback can improve manipulation as well as diagnosis [3] [4].

Prior research has investigated the individual components of telemanipulation systems and teletaction system, including tactile sensors [5] [6] [7] [8], tactile displays [9] [10] [11] [12], and force-reflecting telemanipulators (e.g. [13], [14]), but there is little understanding of the interaction of these components in an integrated telemanipulated teletaction system. In particular, there are critical interactions between force and tactile feedback. To obtain useful tactile information, it is necessary

This work was funded in part by NSF grant EEC-9731748, U.S. Army Medical Research Acquisition Activity under contract DAMD17-01-1-0677, and a NSF graduate research fellowship for the third author.

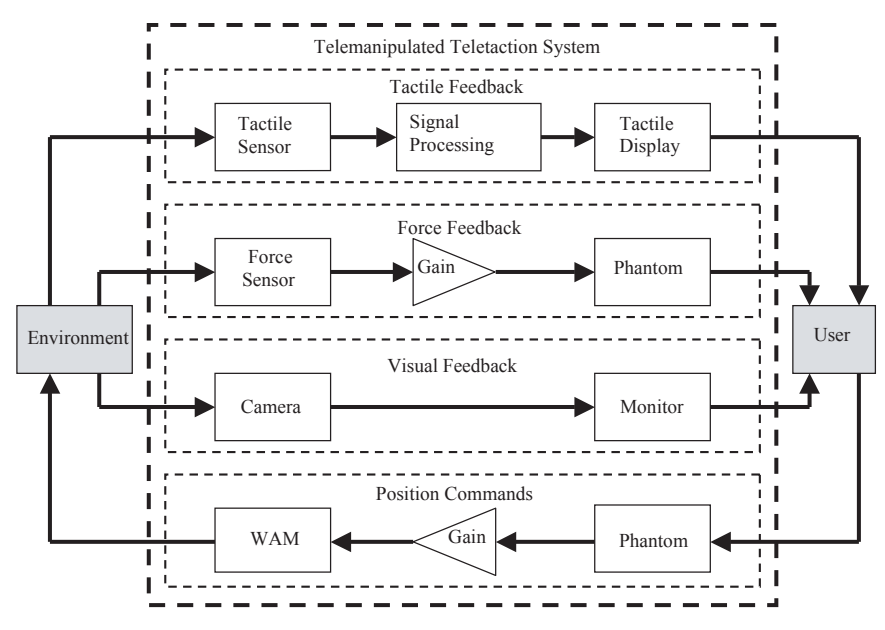

Fig. 1. Telemanipulated teletaction system

to apply an exploration force great enough to stimulate the tactile sensor but small enough to avoid damaging the sensor or the environment.

The main differences between telemanipulation and direct manipulation are the presence of motion scaling, force scaling, and compliance in the telemanipulation system. Given constant motion scaling and compliance in the telemanipulation system, we hypothesize that a change in force scaling results in a change in ability to regulate exploration force. Because of the force requirements of the tactile sensor, changes in the exploration force will affect performance when using a telemanipulated teletaction system versus a directly manipulated teletaction system.

We present an experiment that examines the effect of using a telemanipulated teletaction system versus a directly manipulated teletaction system on finding a rigid lump embedded in a compliant environment. This experiment mimics remote palpation tasks such as breast tumor localization [15] or liver palpation [16]. To investigate the effect of force feedback in telemanipulation on task performance, we varied the force feedback scaling factor between the manipulator and the user. We also conducted a parallel experiment in which subjects directly manipulated the tactile sensor in order to compare telemanipulated and directly manipulated teletaction systems.

\section{Methods And Materials}

The telemanipulated teletaction system used for this experiment consists of a tactile sensor, tactile display, and a force 


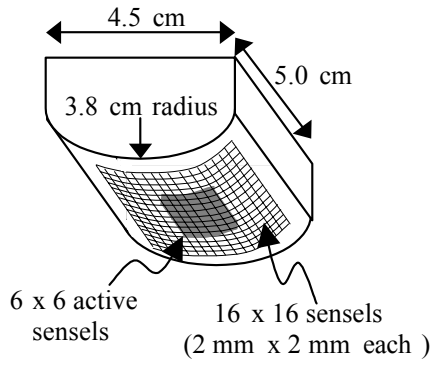

(a)

Fig. 2. Tactile sensor

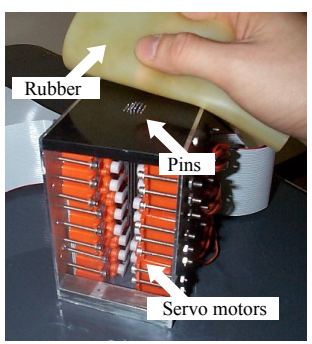

(a) display

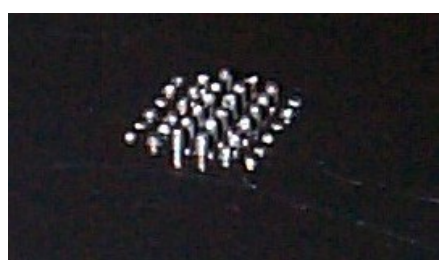

(b) pins
Fig. 3. Tactile display

reflecting telemanipulator (Fig. 1).

\section{A. Teletaction System}

The teletaction system used in this experiment consists of a tactile sensor that measures a two-dimensional pressure profile and a tactile display that recreates shape profiles on the finger pad. The tactile sensor (Pressure Profile Systems, Inc., Los Angeles, CA) measures pressure across a $16 \times 16$ array of pressure sensor elements (sensels) spaced $2 \mathrm{~mm}$ apart (Fig. 2). Each sensel has a pressure resolution of $0.4 \mathrm{kPa}$. We achieve a one-to-one mapping with the tactile display by using only the center $6 \times 6$ sensels. The sensor measures the distribution of pressure across the contact area at a bandwidth of up to $10 \mathrm{~Hz}$.

The tactile display is an array of mechanical pins actuated by commercially available radio controlled (RC) servomotors [10] (Fig. 3). The pins have diameters of $1 \mathrm{~mm}$ and an interpin spacing of $2 \mathrm{~mm}$. They have a maximum displacement of $2 \mathrm{~mm}$ and a vertical resolution of $0.1 \mathrm{~mm}$. A $2 \mathrm{~mm}$ thick piece of silicone rubber (HSII RTV, Dow Corning) was placed on the pins of the tactile display as a spatial low-pass filter [17]. Although the tactile display can run at up to $25 \mathrm{~Hz}$ for small pin movements, the tactile sensor limited the teletaction system bandwidth to $10 \mathrm{~Hz}$.

The pressure profile from the tactile sensor was spatially filtered using a weighted average and then recreated on the tactile display. This filter was necessary to reduce high spatial frequency noise from the tactile sensor. The tactile display pin heights were then set proportional to the pressure data from the tactile sensor. The full signal processing algorithm is given by

$$
D_{i, j}=k[P * A]_{i, j} ; i, j=1, \ldots, 6
$$

where $\mathrm{D}$ is the $6 \times 6$ array of tactile display pin heights, $\mathrm{P}$ is the $6 \times 6$ array of pressure data from the tactile sensor, $k=0.1 \mathrm{~mm} / \mathrm{kPa}$ is a constant that scales measured pressure to display pin height, and $\mathrm{P} * \mathrm{~A}$ denotes convolution of the pressure with $\mathrm{A}$, the noise filtering averaging kernel

$$
A=\left[\begin{array}{ccc}
0.0625 & 0.0625 & 0.0625 \\
0.0625 & 0.5 & 0.0625 \\
0.0625 & 0.0625 & 0.0625
\end{array}\right] \text {. }
$$

Each element in $D$ is then limited to values between 0 and $2 \mathrm{~mm}$ to reflect the capability of the tactile display.

\section{B. Force Reflecting Telemanipulator}

The tactile sensor was mounted in series with a 6-axis forcetorque sensor with a resolution of $0.1 \mathrm{~N}$ (Gamma model, ATI Industrial Automation, Inc, Apex, NC). These sensors were mounted as the end effector of a 6 DOF robot arm (WholeArm Manipulator (WAM), Barrett Technology, Cambridge, MA) (Fig. 4). The WAM was controlled using a $1000 \mathrm{~Hz}$ servo loop running on a DSP (DS1103 PPC, dSPACE, Novi, MI). The position of the WAM was remotely controlled using a Phantom haptic interface device (Model 1.5, Sensable Technologies Inc, Woburn, MA) [18] using scaled Cartesian position mapping (Fig. 5). Such separation of the tactile display and the haptic interface in a telemanipulated lump finding task has been shown not to impede performance [4].

We chose the position scaling factor to be $75 \%$ (a $1.0 \mathrm{~cm}$ displacement of the Phantom produces a $0.75 \mathrm{~cm}$ displacement of the end effector of the WAM). This scaling factor allows the user to scan the entire model within the limits of the Phantom workspace while maintaining safe manipulator speeds. Three of the desired WAM joint angles were computed from the inverse kinematics equations for a 3 DOF planar manipulator (base, elbow, and second wrist joint) given the Cartesian position of the Phantom as the desired position. The second wrist joint was used to maintain the orientation of the sensor with respect to the model, the shoulder roll was locked at $-90^{\circ}$, and the wrist roll was locked at $0^{\circ}$. The desired shoulder pitch angle was computed from the desired depth coordinate using small angle approximations. The desired position was achived using a PID controller producing a stiffness of $1740 \mathrm{~N} / \mathrm{m}$ at the end effector in the direction normal to the plane of exploration. The controller gains were chosen so that the perceived system stiffness was dominated by the remote environment stiffness and not the manipulator compliance, while also maintaing safe torque levels.

Data from the force sensor was filtered using the unweighted average of the previous 20 samples (0.02 s), scaled, and then displayed on the Phantom. Given a typical exploration force of 20-25 N, we selected three force scaling factors $(0.05$, $0.10,0.15)$ to ensure that the output force did not exceed the maximum continuous output of the Phantom (4 N). 


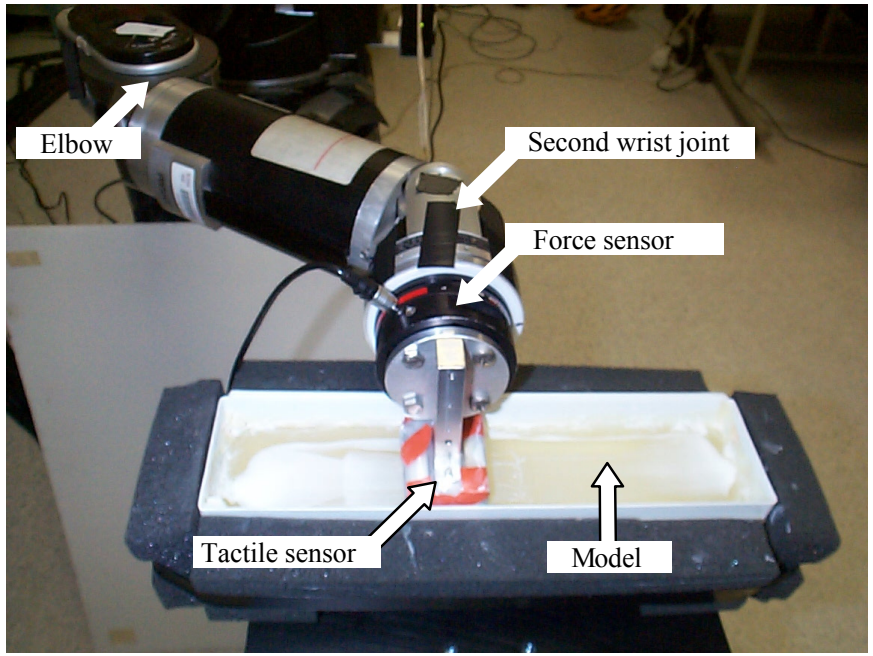

(a) Distal end of remote manipulator with embedded lump model

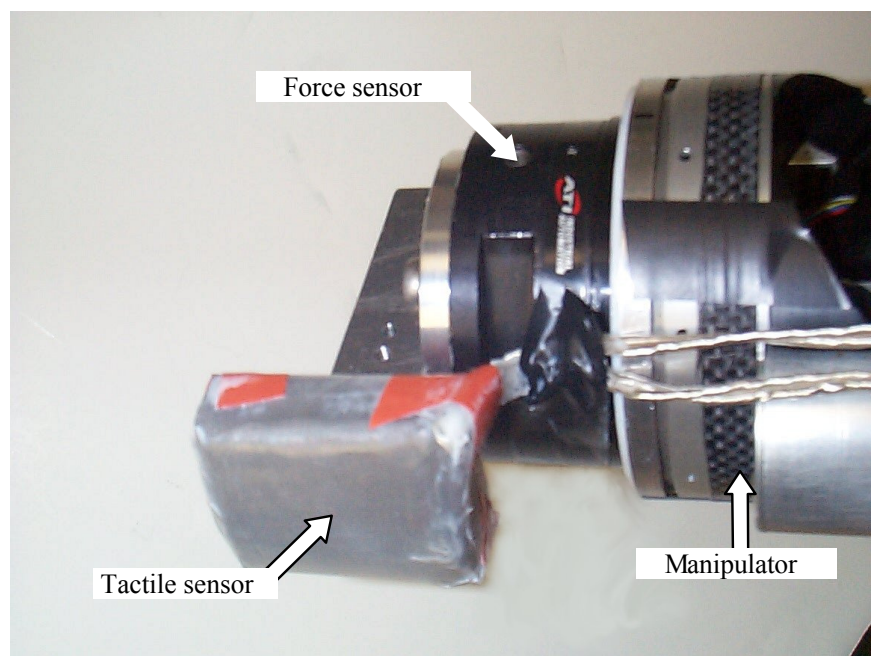

(b) Tactile sensor

Fig. 4. Remote manipulator

Force and position data were recorded at $100 \mathrm{~Hz}$ during the experiment. The one-dimensional position along the model was computed from the forward kinematics of the WAM using the displacement from the starting position in the exploration plane (accuracy: $\pm 2.0 \mathrm{~mm}$ over the length of the model).

\section{Embedded Lump Models}

The three levels of force feedback were compared by having subjects use the telemanipulated teletaction system to locate rigid lumps embedded in constant stiffness elastic models. We constructed four models with a $1.90 \mathrm{~cm}$ diameter rigid acrylic ball on the bottom of a $30.5 \times 7.8 \mathrm{~cm} \times 5.1 \mathrm{~cm}$. The container was filled with silicone rubber (GE Silicones RTV6166) that has a Young's modulus of approximately $15 \mathrm{kPa}$ (Fig. 6). The silicone thickness was chosen to be 1.5 times the diameter of the ball, or $2.86 \mathrm{~cm}$. These parameters allowed users to readily detect a lump with a reasonable exploration force (approx. $15 \mathrm{~N}$ ) and short exploration time (less than $30 \mathrm{~s}$ ).

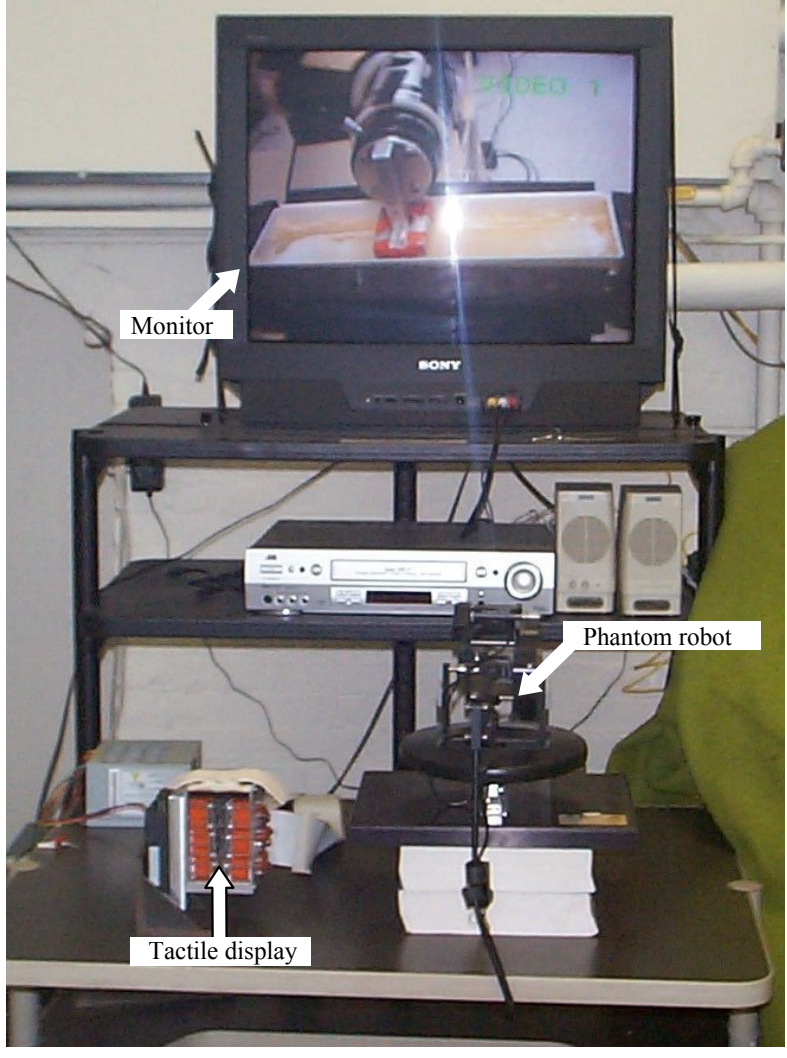

Fig. 5. Teletaction operator work cell

The ball was glued to the bottom of the container in one of four locations, spaced approximately five centimeters apart to minimize memorization of lump location. The width of the container was chosen so that the tactile sensor would fit within the model with less than a centimeter on either side. In this way, subjects need only search in one dimension. A thin layer of latex was placed across the top of each model in order to protect the soft silicone from damage, and hand lotion was used to lubricate the surface so that the sensor could slide across the model with low friction.

During exploration, the model was attached to one end of a lever arm, which was counterbalanced to ensure that the maximum exploration force would not exceed $32 \mathrm{~N}$ to protect the tactile sensor from overload. If the WAM applied a higher force than $32 \mathrm{~N}$, the lever would tip the model down to maintain the maximum contact force.

\section{Experimental Design}

This experiment used a single factor, within subject, repeated measures design with three levels of force feedback. Each level of force feedback was presented 8 times to each subject, for a total of 24 trials. The order of combinations of force feedback and lump position was counterbalanced across trials and across subjects. Six participants between the ages of 20 and 28 volunteered for the experiment. All described themselves as right-handed with no injuries to either hand.

To fully characterize the effect of the telemanipulation 


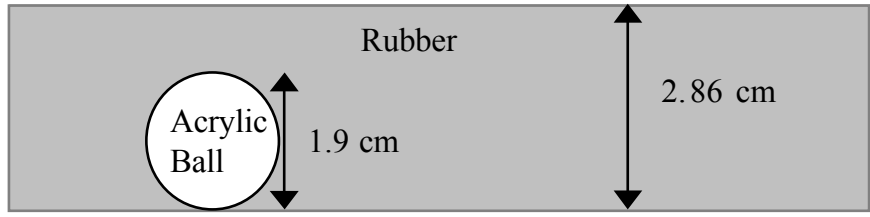

Fig. 6. Cross-section of silicone models

system, a separate set of trials was conducted as part of a parallel experiment, where subjects directly manipulated the tactile sensor (Fig. 7). In addition to the rendering algorithm given by Eqn. (1), this experiment tested two other rendering algorithms. In the second algorithm, a fixed pressure frame was subtracted from each pressure frame received from the tactile sensor. Similar to the second algorithm, the third also subtracted a pressure frame from each frame collected, but the frame that was subtracted depended linearly on the current pressure the user applies. In addition to the embedded lump model thickness of $2.86 \mathrm{~cm}$ used in the telemanipulated experiment, subjects in the directly manipulated experiment explored models of $3.33 \mathrm{~cm}$ and $3.81 \mathrm{~cm}$.

The directly manipulated teletaction experiment used a twofactor, within-subject repeated measures design with algorithms (three levels) and model thickness (three levels). Each combination of algorithm and model thickness was presented ten times to each subject, for a total of 90 trials per subject. The order in which the various combinations of algorithm and model thickness were presented was counterbalanced across trials and across subjects. Fifteen subjects, ranging from ages 20 to 33, volunteered for the experiment for monetary compensation. All subjects described themselves as righthanded and reported no injury to either hand. For detailed description, results, and analysis of the different algorithms used in this experiment, refer to [19]. Only the relevant data from the combination of the first algorithm and the model of $2.86 \mathrm{~cm}$ thickness is presented here for comparison purposes.

In both experiments, we defined a trial as a failure if the final lateral position error was greater than $2.5 \mathrm{~cm}$, half the distance between the placement of the lumps. These trials were discarded from further analysis and noted as trials where the subject did not find the lump.

\section{E. Procedure}

Subjects were instructed that they would be locating hard lumps in soft tissue models by exploring a model using the teletaction system. In the telemanipulated experiment, they were instructed to hold the Phantom stylus with their right hand in a pen grasp and feel the tactile display with their left index finger. In the directly manipulated experiment, subjects were told to hold the tactile sensor with their right hand and feel the tactile display with their left index finger. For each trial, the subject began with the tactile sensor touching the left side of the model's container and then scanned across the one-dimensional model. The subject's primary goal was

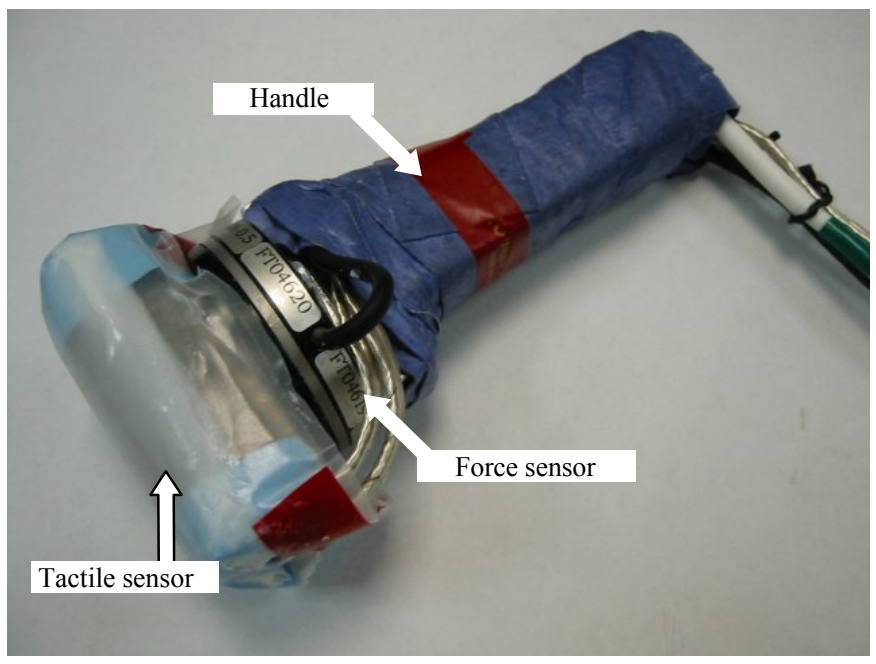

Fig. 7. Tactile sensor used in direct manipulation experiment

to accurately center the tactile sensor directly above the hard lump. Given that they could achieve the primary goal, the secondary goal was to complete this task as fast as possible. The trial stopped when the subject verbally announced that he or she had found the lump.

Although subjects had no time constraints, they were informed that each trial could take up to a minute and the average trial length would be less than thirty seconds. They were given a short break every six trials (telemanipulated experiment) or every nine trials (directly manipulated experiment) and the total experiment length was about an hour. In the telemanipulated experiment, subjects received visual feedback on a monitor from a camera overlooking the model. The visual feedback assisted in positioning the tactile sensor and scanning the model; however, it was not possible to discern the location of the lump in the model from the visual feedback. In the directly manipulated experiment, subjects were blindfolded so their exploration technique would not be affected by observing the thickness of the silicone models. To mask any audio cues, the subjects in both experiments wore earplugs and headphones that played noise in the frequency range of the sounds made by the tactile display.

Before the experiment, subjects were trained to use the teletaction system. They were allowed practice trials until they become comfortable with the operation of the devices. In the telemanipulated experiment, the level of force feedback during the training was counterbalanced across subjects. The subjects were instructed to identify when they exceeded the maximum exploration force from the visual deflection of the mechanical lever-arm and to decrease their exploration force 


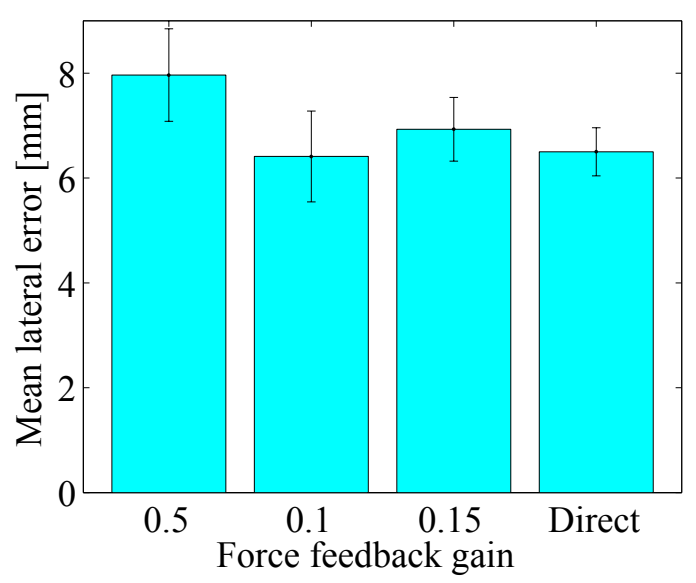

Fig. 8. Mean absolute lateral error. Error bars show standard error.

to a lower level. In the directly manipulated experiment, subjects were trained on a model of medium thickness with a rendering algorithm counterbalanced across subjects. The subjects were then trained to recognize the largest force needed for exploration and to avoid higher forces that might damage the sensor.

\section{RESULTS}

Results for localization lateral position error are shown in Fig. 8. We observed no trend across increasing levels of force feedback gain; subjects localized the lump to within $7 \mathrm{~mm}$ on average. A one factor, three level ANOVA on the lateral position error showed no significant effect $(F(2,88)=1.20$, $p>0.3)$. Further, the mean lateral errors observed for the telemanipulated teletaction case were in the same range as those observed for the directly manipulated teletaction case. $T$-tests between the telemanipulated teletaction cases and the directly manipulated case revealed no significant difference in lateral error between any of the groups $(t(174)=1.89$, $p>0.05$, two-tailed; $t(172)=0.32, p>0.70$, two-tailed; $t(174)=1.08, p>0.25$, two-tailed; direct compared to force feedback gains of $0.05,0.10$, and 0.15 respectively).

Increasing force feedback gain resulted in a significant increase in time taken to localize the lump $(F(2,88)=7.05$, $p<0.001)$. Localization time increased on average from $17 \mathrm{~s}$ to $26 \mathrm{~s}$, an increase of 53\% (Fig. 9). All three telemanipulation localization time means were significantly longer than the directly manipulated teletaction mean of $10 \mathrm{~s}$.

Subjects were unable to find the lump in $3.5 \%$ of the trials, with no significant relationship between number of failures and force feedback gain (Fig. 10) $(F(2,94)=2.04, p>0.1)$. A $t$-test between all telemanipulated teletaction cases and the directly manipulated teletaction case shows the the number of failures did not reach significance $(t(292)=1.02, p>0.30$, two-tailed).

Mean forces applied by the manipulator were calculated for each trial and averaged across subjects. An increase in force feedback gain resulted in a significant decrease in mean applied force (Fig. 11) $(F(2,88)=89.71, p<0.001)$, from a

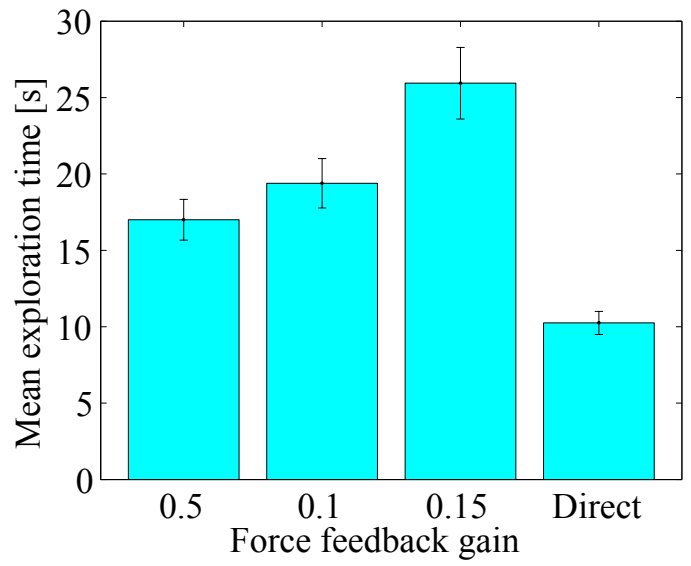

Fig. 9. Mean exploration time. Error bars show standard error.

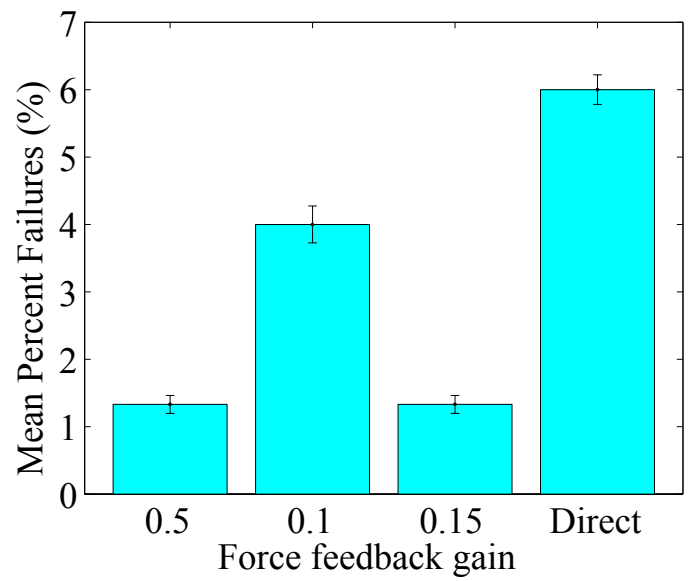

Fig. 10. Mean failure rate. Error bars show standard error.

mean of $19.4 \mathrm{~N}$ to $11.3 \mathrm{~N}$, a decrease of $42 \%$. Note that the mean forces in the telemanipulated case are all less than the mean exploration force of $26 \mathrm{~N}$ during the directly manipulated case. Mean forces one second before the end of the trial were also analyzed to examine the force levels subjects used when positioned over the lump (Fig. 12). Again, an increase in force feedback gain resulted in a significant decrease in final applied force $(F(2,88)=51.31, p<0.001)$, decreasing $36 \%$ from $22 \mathrm{~N}$ to $14 \mathrm{~N}$. Forces in the telemanipulated case were consistently less than the directly manipulated case.

Finally, the mean forces that were applied by the user's hand were found by multiplying the mean exploration force in each force feedback case by the corresponding force feedback gain (Fig. 13). An increase in force feedback gain resulted in a significant increase in the force exerted by the hand $(F(2,88)$ $=78.97, p<0.001$ ), from a mean of $1.0 \mathrm{~N}$ at 0.05 gain to $1.7 \mathrm{~N}$ at 0.15 gain, an increase of $75 \%$.

\section{DISCUSSION}

Our goal was to examine performance in lump localization using a telemanipulated versus directly manipulated teletaction system. We also investigated the effect of force scaling in the 


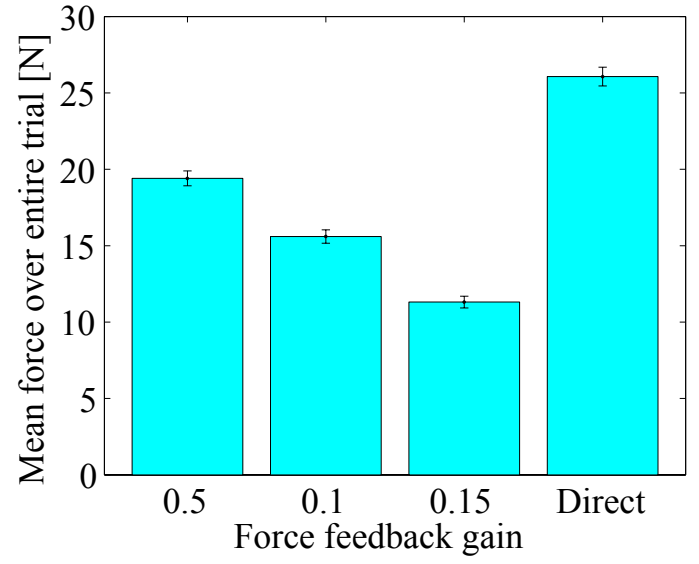

Fig. 11. Mean exploration force. Error bars show standard error.

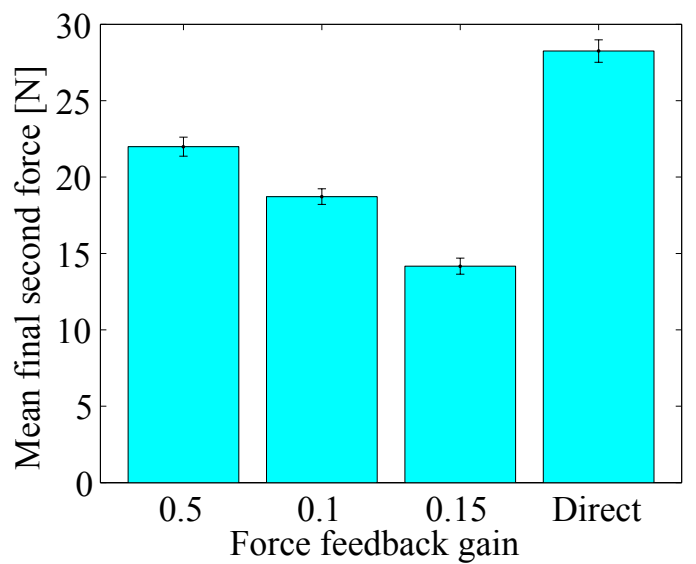

Fig. 12. Mean force applied one second before the end of the trial. Error bars show standard error.

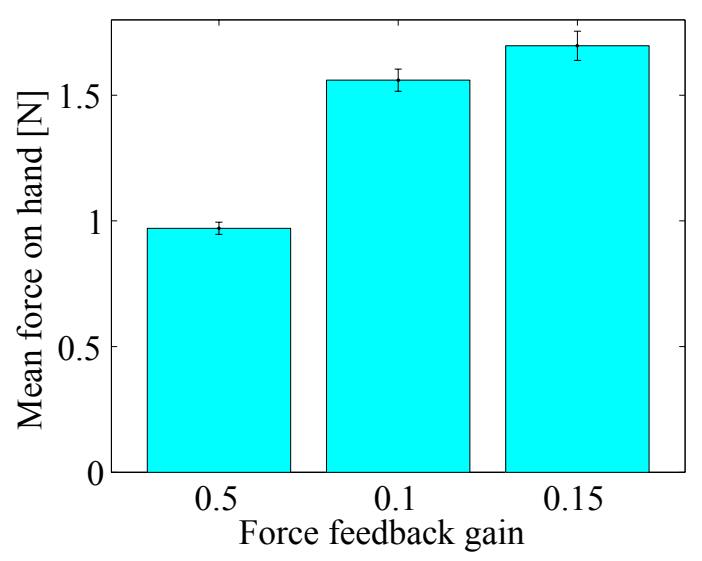

Fig. 13. Mean force applied by hand. Error bars show standard error. telemanipulation system by varying the force feedback gain.

We found that the use of a telemanipulated versus a directly manipulated teletaction system results in no loss of localization accuracy. This result holds for all three levels of force feedback tested. The failure rate is slightly, but not significantly, lower in the telemanipulated versus directly manipulated teletaction system. While accuracy is not significantly affected across experiments, the use of a telemanipulated teletaction system resulted in a significant increase in the time to localize the embedded lump. We also observed in the telemanipulated experiment that forces exerted on the hand increased with increasing force feedback level, but forces exerted by the manipulator on the environment decreased with increasing force feedback level.

The mean exploration time for the telemanipulated teletaction system was significantly longer than the exploration time for the directly manipulated teletaction system (65\% longer for 0.05 gain to $150 \%$ longer for 0.15 gain). A possible explanation for this disparity is that, in the directly manipulated teletaction system, the subjects appeared to use a strategy of maintaining a consistent force sufficient for exploration and could directly control this exploration force. In the telemanipulated teletaction system, however, subjects had to discover what exploration forces were sufficient to obtain enough tactile information due to the varied levels of force feedback. This lack of force control led to a need to re-explore the model.

The mean force applied one second before the end of the exploration, when the subject was localizing the lump, was significantly less in the telemanipulated teletaction system than in the directly manipulated teletaction system (22\% less for 0.05 gain to $50 \%$ less for 0.15 gain). A possible explanation for this observation is that in the directly manipulated teletaction experiment, the subjects explored models of varying thickness where they needed to apply a significantly higher force (approx. 25-30 N) to obtain sufficient tactile information for the thickest models. Given that the subjects were unaware of the model thickness in each trial, they would apply the maximum force necessary for the thickest model, regardless of the relative thickness of the current model.

Given the reduced and varied force feedback level of the telemanipulated teletaction system, it was difficult for the user to apply a consistent exploration force that was sufficient to identify pressure differences (for the models used in the telemanipulated teletaction experiment, greater than approx. 13-15 N). The main information that the subject could base their force control on was the lack of tactile feedback (less than 10-12 $\mathrm{N}$ ) or activating the force limit lever (more than $32 \mathrm{~N}$ ). It also is possible that subjects were able to obtain some additional environment information from the tactile display pin heights, but this is a slight effect that might increase after significant experience. The lack of sufficient exploration force control is visible in the observed trends of mean exploration force and exploration time over the different force feedback levels. In the 0.15 force feedback case, subjects were exploring with a mean force that was insufficient to obtain tactile data, 
compelling them to re-explore the model.

In the telemanipulated teletaction experiment, mean hand force increased with increasing force feedback level, while mean exploration force decreased with increasing force feedback level. To obtain the same tactile information across force feedback levels, we would expect subjects to maintain a constant exploration force across force feedback levels. Mean hand force did increase with increasing force feedback levels, but not enough to maintain a constant exploration force across force feedback levels. This is in accordance with previous research, which has shown that given a task with uncertain impedance, people will try to minimize the energy they extert to accomplish the task [20] [21].

Given the constant motion scaling and compliance in the telemanipulation system, changes to the force feedback gain effectively produce a change in stiffness of the telemanipulation system. This change in the stiffness affects the user's ability to regulate exploration force. Previous research has examined the effects of impedance scaling in standard telemanipulation systems [22] [23], and future work might investigate additional factors that determine impedance (i.e. position scaling and compliance in the manipulator) and their affect on remote palpation.

Maintaining a sufficient exploration force in a telemanipulated teletaction system can be accomplished in several ways. Given a constant stiffness, the user could be trained to recognize the correct exploration force. An alternative is to implement a hybrid control scheme, where the user retains position control, but no longer controls the exploration force; however this presents many safety concerns. An alternative to manipulator controlled exploration force would be to give the user both force and position control, but saturate the exploration force at a desired value. The user would still be able to obtain relevant information about the environment but would not have to worry about exceeding a maximum force threshold. This maximum threshold is crucial in the potential medical applications of remote palpation, where the sensor is exploring areas inside the body.

The results of our experiment led us to conclude that the performance using a teletaction system depends on how well the user can control the exploration force. The quality of the tactile data obtained by the tactile sensor is dependent on the exploration force. If the exploration force is not sufficient to identify pressure differences, the tactile data is insignificant. In order to obtain a significant tactile image, the exploration force must be above some threshold (based on the nature of the task, i.e. relative hardness, size of lump, depth of lump, etc.) but below a level that would be harmful to the sensor or environment. To benefit from the addition of tactile information to a telemanipulation task, we must ensure the desired exploration force is within this range.

\section{REFERENCES}

[1] R. Fearing, G. Moy, and E. Tan, "Some basic issues in teletaction," in Proceedings of International Conference on Robotics and Automation. New York, NY, USA: IEEE, 1997, pp. 3093-9 vol.4.
[2] S. Lederman and R. Klatzky, "Sensing and displaying spatially distributed fingertip forces in haptic interfaces for teleoperator and virtual environment systems," Presence, vol. 8, no. 1, pp. 86-103, 1999.

[3] R. Howe, W. Peine, D. Kantarinis, and J. Son, "Remote palpation technology," IEEE Engineering in Medicine and Biology Magazine, vol. 14, no. 3, pp. 318-23, 1995

[4] W. Peine, "Remote palpation instruments for minimally invasive surgery," Ph.D. dissertation, Harvard University, 1998.

[5] J. Son, E. Monteverde, and R. Howe, "A tactile sensor for localizing transient events in manipulation," in Proceedings of the 1994 IEEE International Conference on Robotics and Automation. Los Alamitos, CA, USA San Diego, CA, USA: IEEE Comput. Soc. Press, 1994, pp. 471-6 vol.1.

[6] R. Fearing, "Tactile sensing mechanisms," International Journal of Robotics Research, vol. 9, no. 3, pp. 3-23, 1990.

[7] B. Gray and R. Fearing, "A surface micromachined microtactile sensor array," in Proceedings of IEEE International Conference on Robotics and Automation. New York, NY, USA Minneapolis, MN, USA: IEEE, 1996, pp. 1-6 vol.1.

[8] R. D. Howe, "Tactile sensing and control of robotic manipulation," Journal of Advanced Robotics, vol. 8, no. 3, pp. 245-261, 1994.

[9] W.-T. Lo, Y. Shen, and Y.-H. Liu, "An integrated tactile feedback system for multifingered robot hands," in Proceedings of RSJ/IEEE International Conference on Intelligent Robots and Systems. Piscataway, NJ, USA Maui, HI, USA: IEEE, 2001, pp. 680-5 vol.2.

[10] C. R. Wagner, S. J. Lederman, and R. D. Howe, "Design and performance of a tactile shape display using rc servomotors," Haptics-e, Submitted, 2003.

[11] D. Caldwell, N. Tsagarakis, and C. Giesler, "An integrated tactile/shear feedback array for stimulation of finger mechanoreceptor," in Proceedings of International Conference on Robotics and Automation. Piscataway, NJ, USA: IEEE, 1999, pp. 287-92 vol.1.

[12] G. Moy, C. Wagner, and R. Fearing, "A compliant tactile display for teletaction," in Proceedings 2000 ICRA. IEEE International Conference on Robotics and Automation. Piscataway, NJ, USA: IEEE, 2000, pp. 3409-15 vol.4.

[13] T. B. Sheridan, Telerobotics, automation, and human supervisory control. Cambridge, Mass.: MIT Press, 1992.

[14] B. Hannaford and P. Fiorini, "A detailed model of bi-lateral teleoperation," in Proceedings of the 1988 IEEE International Conference on Systems, Man, and Cybernetics (IEEE Cat. No.88CH2556-9). Beijing, China: Int. Acad. Publishers, 1988, pp. 117-21 vol.1.

[15] H. S. Pennypacker and C. A. Pilgrim, "Achieving competence in clinical breast examination," Nurse Pract Forum, vol. 4, no. 2, pp. 85-90, 1993.

[16] C. Naylor, "Physical examination of the liver," JAMA, vol. 271, no. 23, pp. 1859-65, 1994.

[17] J. M. Lee, C. R. Wagner, S. J. Lederman, and R. D. Howe, "Spatial low pass filters for pin actuated tactile displays," in 11th Annual International Symposium on Haptic Interfaces for Virtual Environment and Teleoperator Systems, IEEE, Ed. Los Angeles, CA: IEEE, 2003.

[18] M. C. Cavusoglu, D. Feygin, and F. Tendick, "A critical study of the mechanical and electrical properties of the phantom haptic interface and improvements for high performance control," Presence: Teleoperators and Virtual Environments, vol. 11, no. 6, pp. pp. 555 - 568, 2002.

[19] C. K. L. Lau, C. R. Wagner, and R. D. Howe, "Algorithms for tactile rendering in compliant environments," in 12th Annual International Symposium on Haptic Interfaces for Virtual Environment and Teleoperator Systems, IEEE, Ed., New Orleans, LA, 2004.

[20] R. Johansson and G. Westling, "Coordinated isometric muscle commands adequately and erroneously programmed for the weight during lifting task with precision grip," Experimental Brain Research, vol. 71, no. 1, pp. 59-71, 1988.

[21] R. Osu, D. Franklin, H. Kato, H. Gomi, K. Domen, T. Yoshioka, and M. Kawato, "Short- and long-term changes in joint co-contraction associated with motor learning as revealed from surface emg," Journal of Neurophysiology, vol. 88, no. 2, pp. 991-1004, 2002.

[22] J. Colgate, "Power and impedance scaling in bilateral manipulation," in IEEE Intl. Conf on Robotics and Automation, Sacramento, CA, 1991, pp. 2292-2297.

[23] H. Tokashiki, P. Akella, and K. Tanie, "Scaled bilateral telemanipulation: an experimental investigation of scaling laws," Proceedings of the SPIE - The International Society for Optical Engineering, vol. 2351, pp. 21424, 1994. 